\title{
Efficacy and tolerability of transdermal granisetron for the control of chemotherapy-induced nausea and vomiting associated with moderately and highly emetogenic multi-day chemotherapy: a randomized, double-blind, phase III study
}

\author{
Ralph V. Boccia • Lucio N. Gordan • Gemma Clark • \\ Julian D. Howell • Steven M. Grunberg • \\ on behalf of the Sancuso Study Group \\ Received: 14 June 2010 / Accepted: 16 August 2010/Published online: 12 September 2010 \\ (C) The Author(s) 2010. This article is published with open access at Springerlink.com
}

\begin{abstract}
Purpose A novel transdermal formulation of granisetron (the granisetron transdermal delivery system (GTDS)) has been developed to deliver granisetron continuously over 7 days. This double-blind, phase III, non-inferiority study compared the efficacy and tolerability of the GTDS to daily
\end{abstract}

The data in this paper were previously presented at the MASCC 20th International Annual Symposium, St Gallen, Switzerland, 27-30 June 2007, no. 18: Phase III trial of transdermal granisetron patch

(Sancuso) compared with oral granisetron in the management of chemotherapy-induced nausea and vomiting (CINV) by Steven M Grunberg, Nashat Y Gabrial, and Gemma Clark (poster presentation).

Study number: $392 \mathrm{MD} / 15 / \mathrm{C}$

Clinicaltrials.gov identifier: NCT00273468

Electronic supplementary material The online version of this article (doi:10.1007/s00520-010-0990-y) contains supplementary material, which is available to authorized users.

\section{R. V. Boccia $(\bowtie)$}

Center for Cancer and Blood Disorders,

6420 Rockledge Dr., No. 4100,

Bethesda, MD 20817, USA

e-mail: rboccia@ccbdmd.com

\section{N. Gordan}

Clinical Research Department,

Gainesville Hematology-Oncology Associates,

Gainesville, FL, USA

\section{G. Clark · J. D. Howell}

Clinical Development, ProStrakan Inc,

Bedminster, NJ, USA

S. M. Grunberg

Division of Hematology/Oncology, University of Vermont,

Burlington, VT, USA oral granisetron for the control of chemotherapy-induced nausea and vomiting (CINV).

Patients and methods Six hundred forty-one patients were randomized to oral (2 $\mathrm{mg}$ /day, 3-5 days) or transdermal granisetron (one GTDS patch, 7 days), before receiving multi-day chemotherapy. The primary endpoint was complete control of CINV (no vomiting/retching, no more than mild nausea, no rescue medication) from chemotherapy initiation until $24 \mathrm{~h}$ after final administration. The prespecified non-inferiority margin was $15 \%$.

Results Five hundred eighty-two patients were included in the per protocol analysis. The GTDS displayed noninferiority to oral granisetron: complete control was achieved by $60 \%$ of patients in the GTDS group, and $65 \%$ in the oral granisetron group (treatment difference, $-5 \%$; $95 \%$ confidence interval, $-13-3)$. Both treatments were well tolerated, the most common adverse event being constipation.

Conclusions The GTDS provides effective, well-tolerated control of CINV associated with moderately or highly emetogenic multi-day chemotherapy. It offers a convenient alternative route for delivering granisetron for up to 7 days that is as effective as oral granisetron.

Keywords Cancer. Chemotherapy-induced nausea and vomiting (CINV) · Granisetron · Multi-day · Transdermal

\section{Introduction}

Multi-day chemotherapy is associated with a substantial risk of chemotherapy-induced nausea and vomiting (CINV) 
[1], yet remains the mainstay of treatment strategies for many cancers $[2,3]$. Prophylaxis of CINV associated with multi-day chemotherapy is, therefore, a key clinical goal and, although relatively under-represented in the literature $[4,5]$, an important area of research.

The management of CINV in patients receiving multiday chemotherapy presents additional challenges, compared to single-day chemotherapy. The pathophysiology of CINV in this setting is complicated by the overlap of acute and delayed nausea and vomiting, making meaningful distinction between these two parameters difficult and requiring use of overall protection from nausea and vomiting as a primary endpoint [2]. Repeated administration of medications over several days can give rise to peaks and troughs in plasma drug levels $[6,7]$. In addition, such treatment may require a high tablet burden and complex dosing regimen, with potential detrimental effects on treatment adherence [8]. Delivery of antiemetic therapy from a transdermal system may help to reduce these obstacles to CINV control.

Current guidelines for the management of multi-day CINV recommend 5-hydroxytryptamine subtype $3\left(5-\mathrm{HT}_{3}\right)$ receptor antagonists (RAs) as a basis for treatment, to which corticosteroids, with or without a neurokinin-1 (NK-1) RA, may be added [5, 9, 10]. The commonly used $5-\mathrm{HT}_{3}$ RA granisetron is currently available for oral and intravenous administration. A new formulation, the granisetron transdermal delivery system (GTDS; Sancuso ${ }^{\circledR}$, ProStrakan Pharmaceuticals), has been developed to provide extended release of granisetron over 7 days. The GTDS patch is an $8 \times 6-\mathrm{cm}$ clear plastic-backed patch with an adhesive layer containing $34.3 \mathrm{mg}$ of granisetron. Pharmacokinetic evaluation of the GTDS has indicated that it provides continuous delivery of granisetron over 7 days, providing exposure similar to an oral dose of $2 \mathrm{mg}$ per day [11]. Transdermal delivery offers a convenient non-invasive option for sustained antiemetic administration and could enable continuous antiemetic prophylaxis throughout a multi-day chemotherapy regimen.

This study compares the efficacy and tolerability of the GTDS with those of oral granisetron for controlling CINV in patients receiving moderately emetogenic chemotherapy (MEC) or highly emetogenic chemotherapy (HEC) regimens for 3-5 days.

\section{Methods}

\section{Patients}

Adult cancer patients $(\geq 15,16$, or 18 years, dependent on local regulations) assigned to receive the first cycle of a new multi-day MEC or HEC regimen were eligible for enrolment in the study. An emetogenicity classification for multi-day chemotherapy was devised based on the singleagent Hesketh level for the most emetogenic agent, modified when additional emetogenic agents were included in the regimen (online resource, section 1) [12]. MEC and HEC regimens were defined as those with maximum emetogenicity levels of 3-4 and $>4$, respectively, for a 3-5-day period.

Inclusion criteria included an Eastern Cooperative Oncology Group (ECOG) status $\leq 2$ and a life expectancy of $\geq 3$ months. The primary exclusion criteria included: hypersensitivity to adhesive plasters; contraindications to 5-HT 3 RAs; participation in any previous clinical study with the GTDS; any cause for nausea and vomiting other than CINV, including radiotherapy within 1 week of entry; any retching, nausea, or uncontrolled vomiting within $72 \mathrm{~h}$ before chemotherapy initiation; clinically relevant abnormal ECG parameters; and/or baseline QTc prolongation.

Study design

This was a randomized, active control, double-blind, parallelgroup, phase III study, conducted at 60 centers in Europe, India, Mexico, and the USA. The primary objective was to demonstrate non-inferiority of GTDS efficacy compared to oral granisetron, in patients receiving MEC or HEC. Secondary objectives included assessment of the safety, tolerability, and adhesive properties of the GTDS.

Following a 7-day screening period, patients were randomized in a 1:1 ratio using a telephone-based randomization system to receive either a GTDS patch and placebo capsules or a placebo patch and active capsules $(2 \mathrm{mg}$ granisetron). Active and placebo medications were identical in appearance to ensure blinding. Patients were allocated to treatment in a random permuted block design, 1-2 days before initiation of chemotherapy. Stratification was based on factors known to affect CINV, including gender, planned chemotherapy regimen and duration, and chemotherapy naivety. Stratification of chemotherapy regimens was based on the inclusion or exclusion of cisplatin in the regimen; non-cisplatin regimens were further divided based on the planned inclusion of corticosteroids as part of the antiemetic regimen. The use of corticosteroids with cisplatin regimens was not included in the stratification, as it was anticipated that the vast majority of patients receiving cisplatin would also receive corticosteroids as part of the regimen. The use of corticosteroids in this group was recorded for confirmation.

Due to the gradual dermal penetration of granisetron from the transdermal system, patches were applied to the upper arm 24-48 $\mathrm{h}$ before the start of chemotherapy, by the investigator or the patient, and left in place for 7 days. Capsules were administered $1 \mathrm{~h}$ before each day's administration of chemotherapy (online resource, section 1). Patches 
were left in place for a full 7 days in all patients in order to increase the amount of safety data collected. Corticosteroids were permitted at the discretion of the investigator, as prophylaxis, and started before chemotherapy. The use of other concomitant therapies was permitted, excluding concomitant radiotherapy; medication to control the symptoms of a brain tumor, brain metastasis, or seizure disorder; SSRI antidepressants (unless a stable dose for the duration of the study); drugs known to increase the QTc interval; any other investigational drug; and any NK-1 RA, dopamine RA, or $5-\mathrm{HT}_{3} \mathrm{RA}$.

Prescription of rescue medication not known to cause QTc prolongation was permitted at the discretion of the investigator, in accordance with the site's standard of care. Increased use of corticosteroids above that planned in the chemotherapy regimen was recorded as rescue medication.

All patients provided written informed consent before enrolment into the study. The study protocol was approved by the Ethics Committee/Institutional Review Board at each participating center and was conducted in accordance with the Declaration of Helsinki.

\section{Efficacy parameters}

The primary efficacy endpoint was the percentage of patients achieving complete control of CINV (CC; no vomiting and/or retching, no more than mild nausea, and no use of rescue medication) from the first administration until $24 \mathrm{~h}$ after the last administration of chemotherapy (the primary endpoint evaluation period (PEEP); online resource, Figure S1). Nausea and vomiting were assessed using patient diaries, completed on a daily basis. Patients reported the presence and severity of nausea on a four-point scale (none, mild, moderate, severe) and the presence and severity of vomiting on a five-point scale (none, mild, moderate, severe, very severe).

Exploratory analyses of the primary endpoint included analysis in predefined subgroups based on randomization stratification variables. Secondary efficacy endpoints included the time to failure of CC; the percentage of patients failing CC due to nausea, vomiting, or receipt of rescue medication; the percentage of patients achieving complete response (CR; no vomiting and/or retching, no rescue medication) or total control (TC; no vomiting and/or retching, no nausea, no rescue medication) during the PEEP (overall and by day); patients' global satisfaction with antiemetic therapy (assessed using a $10-\mathrm{cm}$ visual analog scale (VAS) at the time of patch removal); and the percentage adhesion of the patch over the application period.

Study visits and evaluations

Patients were assessed for eligibility 4-12 days before initiation of chemotherapy. Assessments of efficacy, toler- ability, and safety variables were performed throughout the study period, including a 14-day follow-up after patch removal (online resource, Figure S1). Emesis control, rescue medication, and patch adhesion were recorded using patient diary cards, distributed at visit 1 .

\section{Statistical analysis}

The primary efficacy endpoint was assessed using a noninferiority model, with a non-inferiority margin $(\Delta)$ of $15 \%$. This non-inferiority margin was based on previous non-inferiority studies of 5- $\mathrm{HT}_{3} \mathrm{RA}$ antiemetics in CINV $[13,14]$, and is consistent with FDA and EU reviews of alternative $5-\mathrm{HT}_{3} \mathrm{RAs}[15,16]$, to which granisetron has demonstrated equivalence [17]. Assuming 50\% CC with oral granisetron and a difference between treatment groups of $\leq 15 \%$ (the non-inferiority margin), 576 patients were required to ensure $90 \%$ power. Using a predicted drop-out rate of $9 \%$, randomization of 630 patients was planned.

Non-inferiority of the GTDS compared to oral granisetron was assessed by constructing a point estimate of the difference in emetic control between treatments. Non-inferiority was accepted if the lower limit of the two-sided $95 \%$ confidence interval (CI) of this point estimate was greater than $-\Delta$. Differences between treatments, and corresponding CIs, were evaluated using a logistic regression model, adjusting for study treatment and randomization stratification variables.

Inferiority limits were not prespecified for exploratory analyses. Statistical significance of differences in secondary endpoints was calculated using Cox's proportional hazard model (time to events), logistic regression (CR and TC), and analysis of covariance (patients' global satisfaction).

Statistical analyses were performed in the safety set (SS; all patients who received at least one dose of study treatment), the full analysis set (FAS; all SS patients who had $\geq 1$ efficacy assessment), and the per protocol set (PPS; all FAS patients who had no protocol violations that directly impinged on or affected the primary endpoint, defined before unblinding). As this was a non-inferiority study, the primary efficacy analysis was performed in the PPS. This is in line with guidelines from the International Conference on Harmonisation, and aims to avoid the tendency towards equivalence associated with intent-totreat analyses [18]. The results were validated using additional analyses in the FAS [18].

\section{Results}

Patient characteristics

The study was carried out between January and October 2006. Of 715 patients assessed for eligibility, 641 were 
randomized to receive the GTDS $(n=318)$ or oral granisetron $(n=323)$. Of these patients, 637 were in the SS, 621 were in the FAS, and 582 were in the PPS; reasons for withdrawal were similar between the two treatment groups (Fig. 1). Baseline demographic and medical characteristics, including stratification variables, were comparable between the two treatment groups (Table 1). Baseline characteristics in the SS and PPS were similar to the FAS (data not shown). Prophylactic use of corticosteroids was well balanced between the two arms (GTDS group, 61.0\%; placebo group, 61.7\%). In the FAS, $71 \%$ of GTDS patients and $72 \%$ of oral granisetron patients received regimens containing one or more days of cisplatin.

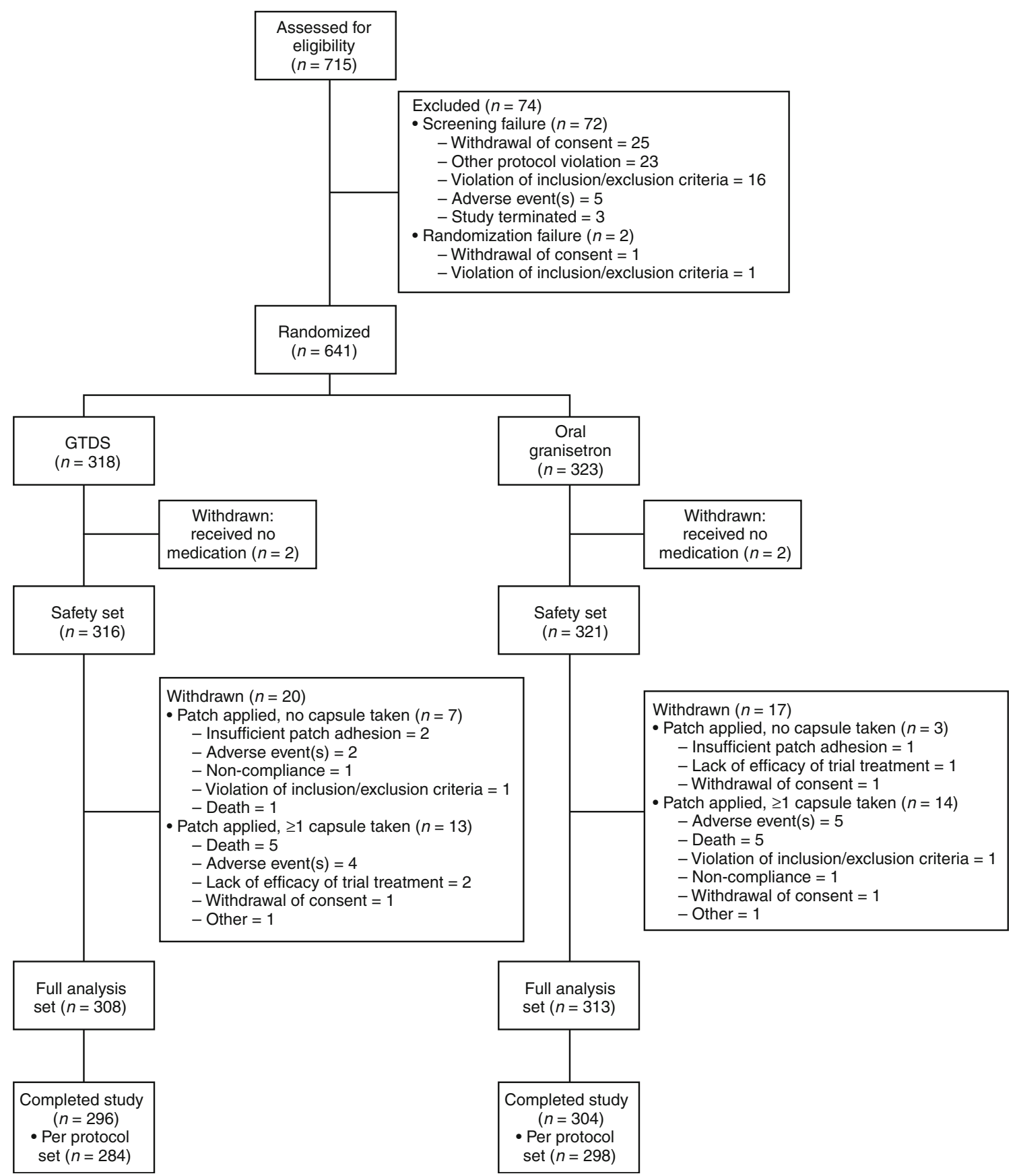

Fig. 1 Flow of participants through the study SS, all patients who received $\geq 1$ dose of study treatment. FAS, all SS patients who had $\geq 1$ efficacy assessment after the start of chemotherapy. PPS, all FAS patients without protocol violations that directly impinged on or affected the primary endpoint. Protocol violations leading to exclusion from the PPS included changes in the planned chemotherapy regimen, receipt of banned concomitant medications, $>50 \%$ patch detachment and incorrect study drug administration 
Table 1 Patient baseline characteristics and therapy history (FAS)

GTDS $(N=308) \quad$ Oral granisetron $(N=313)$

Age
Mean $\pm \mathrm{SD}$ (years)
$\leq 40$ years
$>40$ years

Physical characteristics

Weight, mean \pm SD (kg)

Height, mean \pm SD $(\mathrm{cm})$

BMI, mean $\pm \mathrm{SD}\left(\mathrm{kg} / \mathrm{m}^{2}\right)$

Gender $^{\mathrm{a}}$

Male

Female

Ethnic origin

White

Asian

Hispanic/Latino

Smoking status

Smoker

Ex-smoker

Never smoked

Current alcohol consumption

None

Monthly

Weekly

Daily

ECOG performance status

0

1

2

Metastatic disease

Yes

History of radiotherapy

Yes

Chemotherapy regimen ${ }^{\mathrm{a}}$

Cisplatin

Non-cisplatin + corticosteroids

Non-cisplatin - corticosteroids

Duration of chemotherapy ${ }^{\mathrm{a}}$

$\leq 3$ days

$4 / 5$ days

Chemotherapy naivety ${ }^{\mathrm{a}}$

Naïve

Non-naïve

Unless otherwise stated, data show percentage of patients

$S D$ standard deviation

${ }^{a}$ Gender, planned chemotherapy regimen and chemotherapy naivety were used as stratification variables for randomization. Stratification of chemotherapy regimens divided regimens into cisplatin and non-cisplatin chemotherapy. Non-cisplatin regimens were further divided based on whether corticosteroids were pre-planned for inclusion as antiemetics. The use of corticosteroids concomitantly with cisplatin was not included in the stratification, but was well balanced between treatment groups (data not shown)
Primary efficacy analysis

The study met its predefined primary endpoint. The lower limit of the $95 \%$ CI of the difference between the treatments was greater than $-15 \%$, indicating that the GTDS was noninferior relative to oral granisetron. The number of patients in the PPS who achieved CC of CINV during the PEEP was $193(65 \%)$ in the oral granisetron group and $171(60 \%)$ in the GTDS group (Table 2). This equates to an estimated difference in percentage $\mathrm{CC}$ between the groups of $5 \%$ (95\% CI, $-13-3$, Table 2).

Secondary and exploratory efficacy analyses

Examination of $\mathrm{CC}$ in predefined subgroups of the PPS, based on known CINV risk factors, showed no significant differences between the GTDS and oral granisetron in any subgroup (Fig. 2). This indicates that the results of the primary efficacy analysis are consistent across these predefined subgroups. Additionally, analysis of CC in the FAS gave similar results to those obtained in the PPS (Table 2). The results of these exploratory analyses, therefore, support the conclusion of non-inferiority of the GTDS relative to oral granisetron.

The percentage of patients in the PPS who achieved $\mathrm{CR}$ and TC during the PEEP is shown in Table 2. No significant differences were observed in the percentages of patients achieving $\mathrm{CR}$ and TC in the two treatment groups. For those patients who did not achieve CC (113 and 105 treatment failures in the GTDS and oral groups, respectively), the time to $\mathrm{CC}$ failure was similar for the two groups (online resource, section 2). In patients receiving 3- and 5-day chemotherapy regimens, $\mathrm{CC}$ and $\mathrm{CR}$ were similar between the GTDS and oral granisetron treatment groups on all days of therapy (online resource, section 2, Figure S2).

Patients' global satisfaction with antiemetic therapy was high with both treatments. The mean ( \pm standard deviation) VAS score for patients in the FAS was $8.19 \mathrm{~cm}( \pm 2.21)$ for GTDS-treated patients and $8.24 \mathrm{~cm}( \pm 2.13)$ for oral granisetron patients, $p=0.887$. There was no significant difference between the two groups.

Patch adhesion

The adhesive properties of the active and placebo patches were assessed in the FAS. Of 308 patients receiving the GTDS, 198 (64\%) had $\geq 90 \%$ adhesion and 277 (90\%) had $\geq 75 \%$ adhesion. Two patients (1\%) had a completely detached patch. Of the 313 patients who received a placebo patch, 244 (78\%) had $\geq 90 \%$ adhesion and 299 (96\%) had $\geq 75 \%$ adhesion. Three patients (1\%) had a completely detached patch. 
Table 2 Complete control, complete response and total control of CINV in the PPS and FAS

$n$ number of patients achieving response, $N$ total number of patients in the treatment group

\begin{tabular}{|c|c|c|c|c|c|c|}
\hline & \multicolumn{2}{|l|}{ GTDS } & \multicolumn{2}{|c|}{ Oral granisetron } & \multirow[t]{2}{*}{ Difference $(\%)$} & \multirow[t]{2}{*}{$95 \% \mathrm{CI}$} \\
\hline & $n / N$ & $\%$ & $n / N$ & $\%$ & & \\
\hline \multicolumn{7}{|l|}{ Per protocol set } \\
\hline Complete control & $171 / 284$ & 60 & $193 / 298$ & 65 & -4.9 & $-12.9-3.1$ \\
\hline Complete response & $176 / 284$ & 62 & $203 / 298$ & 68 & -6.6 & $-14.4-1.3$ \\
\hline Total control & $158 / 284$ & 56 & $177 / 298$ & 59 & -3.8 & $-11.8-4.3$ \\
\hline \multicolumn{7}{|l|}{ Full analysis set } \\
\hline Complete control & $185 / 308$ & 60 & $205 / 313$ & 65 & -5.8 & $-13.5-2.0$ \\
\hline
\end{tabular}

\section{Tolerability}

A total of 637 patients were included in the SS, of whom 254 experienced adverse events (GTDS, 128/316; oral granisetron, 126/321). The majority of treatment-emergent adverse events (TEAEs) were of mild or moderate severity, and the incidence of TEAEs was comparable between the GTDS (41\%) and oral granisetron (39\%) groups. The majority of TEAEs were considered unrelated to the study drugs. Study drug-related TEAEs that occurred throughout the study are summarized in Table 3 . The most common study drug-related TEAE in both treatment groups was constipation, which was reported more frequently by patients receiving the GTDS than oral granisetron $(7 \%$ and $3 \%$, respectively). In contrast, headache was reported more frequently by patients receiving oral granisetron than the GTDS (2.5\% and $0.3 \%$, respectively).
Local skin tolerability of the patch was good. Application site pruritus was reported in two cases (both in the GTDS group), of which only one was deemed to be study drug related.

Serious TEAEs were reported by a total of 47 patients, of which five were considered study drug related (oral granisetron group: QTc prolongation, three and toxic megacolon, one; GTDS group: constipation, one). There were 15 deaths in the study population (seven GTDS and eight oral granisetron patients), but only one (toxic megacolon, oral granisetron group) was regarded as related to study treatment. Seven patients in each group withdrew from the study due to adverse events.

The ECGs of all patients were subjected to blinded expert review. In 468 patients for whom full ECG data were available, no clinically significant ECG morphology changes were observed for either treatment, and no cases of QTc prolongation were identified in the GTDS group.

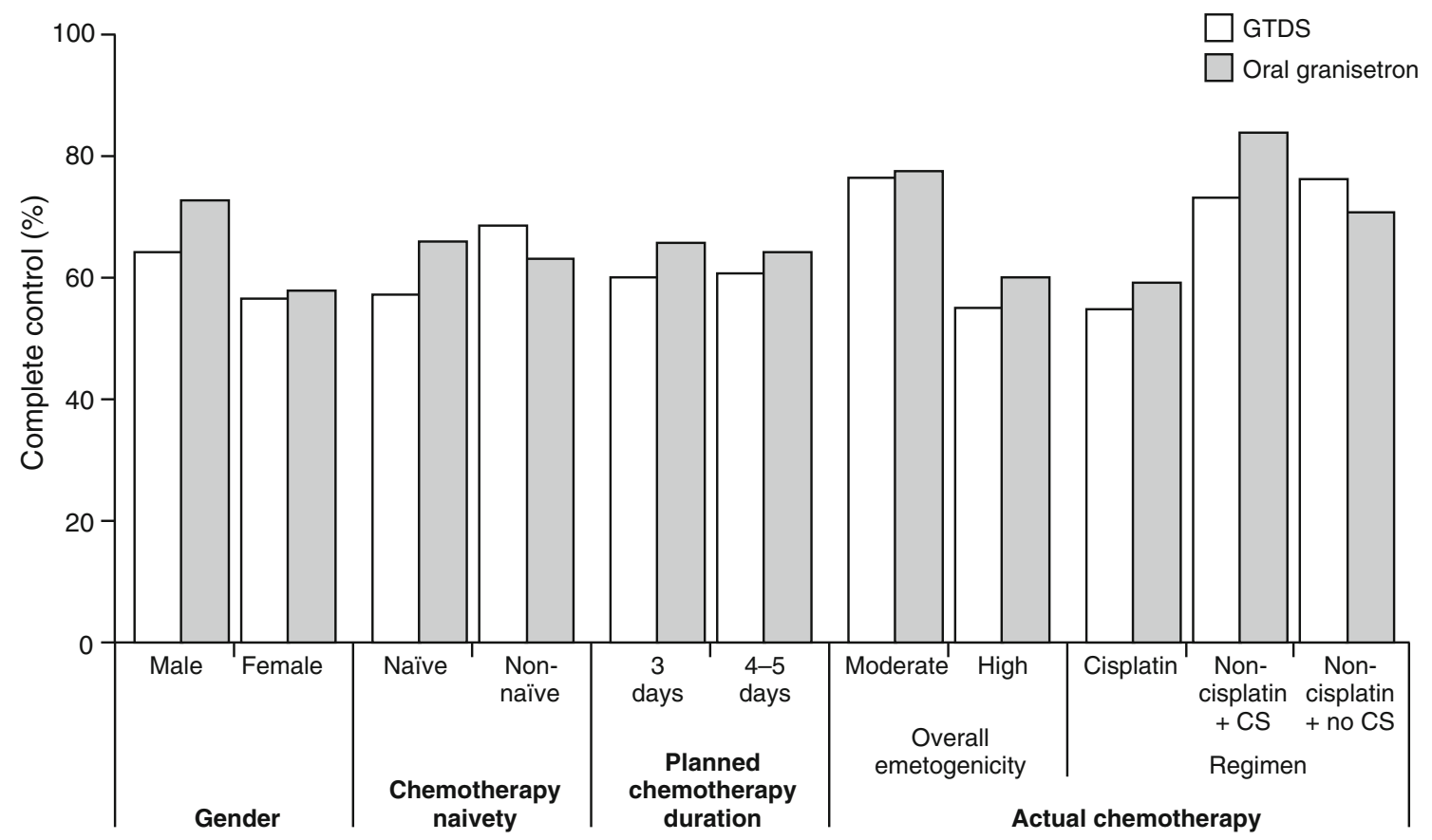

Fig. 2 Complete control of CINV in predefined subgroups of the PPS. CS corticosteroids 
Table 3 Study drug-related treatment-emergent adverse events in the GTDS and oral granisetron groups (SS) $n$ number of patients with adverse event, $N$ total number of patients in the treatment group

\begin{tabular}{|c|c|c|c|c|}
\hline & \multirow{2}{*}{\multicolumn{2}{|c|}{$\begin{array}{l}\text { GTDS } \\
(N=316)\end{array}$}} & \multirow{2}{*}{\multicolumn{2}{|c|}{$\begin{array}{l}\text { Oral granisetron } \\
(N=321)\end{array}$}} \\
\hline & & & & \\
\hline & $n$ & $\%$ & $n$ & $\%$ \\
\hline Gastrointestinal disorders & 22 & 7 & 12 & 3.7 \\
\hline Constipation & 21 & 6.6 & 10 & 3.1 \\
\hline Abdominal pain & 0 & 0 & 1 & 0.3 \\
\hline Irritable bowel syndrome & 0 & 0 & 1 & 0.3 \\
\hline Megacolon & 0 & 0 & 1 & 0.3 \\
\hline Nausea & 1 & 0.3 & 0 & 0 \\
\hline General disorders and administrative site conditions & 1 & 0.3 & 0 & 0 \\
\hline Application site pruritus & 1 & 0.3 & 0 & 0 \\
\hline Metabolism and nutrition disorders & 2 & 0.6 & 1 & 0.3 \\
\hline Anorexia & 1 & 0.3 & 1 & 0.3 \\
\hline Decreased appetite & 1 & 0.3 & 0 & 0 \\
\hline Nervous system disorders & 1 & 0.3 & 8 & 2.5 \\
\hline Headache & 1 & 0.3 & 8 & 2.5 \\
\hline Vascular disorders & 1 & 0.3 & 0 & 0 \\
\hline Flushing & 1 & 0.3 & 0 & 0 \\
\hline Investigations & 1 & 0.3 & 3 & 0.9 \\
\hline ECG QT corrected interval prolonged & 0 & 0 & 3 & 0.9 \\
\hline Weight decreased & 1 & 0.3 & 0 & 0 \\
\hline
\end{tabular}

\section{Discussion}

The current randomized trial represents the first direct comparison of the efficacy of a transdermal 5- $\mathrm{HT}_{3} \mathrm{RA}$ with the oral dose form. The results of the primary efficacy assessments indicated that the GTDS was non-inferior to oral granisetron in the control of CINV in patients receiving multi-day chemotherapy. Similar results were obtained in exploratory analyses of the primary efficacy variable and in secondary efficacy endpoints.

Based on the prespecified non-inferiority margin of $15 \%$, the results of this study indicate that the GTDS was noninferior to oral granisetron. A non-inferiority study design was appropriate in this case, as both treatment arms used the same active drug, and the GTDS was designed to give equivalent systemic exposure to the established comparator.

The exploratory subgroup analyses and secondary endpoints further support the conclusion of non-inferiority. Importantly, efficacy was maintained when all grades of nausea were included in the analysis, for both MEC and HEC, and in patients with known CINV risk factors (online resource, section 3). In addition, the GTDS maintained CINV control from day to day, and was not inferior to oral granisetron on any day of the PEEP (online resource, Figure S2). The rates of CINV control observed on individual days were similar to previously reported data with granisetron (Kytril ${ }^{\circledR}$ Product Information 2005).
The addition of corticosteroids to $5-\mathrm{HT}_{3} \mathrm{RA}$ antiemetic therapy improves emetic control, and is supported by current guidelines [9]. The use of corticosteroids was allowed in this study but not standardized, reflecting the community standard of care across the countries included in the study; however, steroid use was balanced between treatment groups.

The adverse event profile of the GTDS observed in this study indicates that it was well tolerated in cancer patients, with adverse events reflective of those previously observed for granisetron (Table 3). Skin tolerability of the patch was good, as application site reactions were reported only once by GTDS patients and did not cause any patients to withdraw. Interestingly, the incidence of headache was lower in the GTDS group than the oral granisetron group. Constipation was reported more frequently in the GTDS group than the oral granisetron group; however, all GTDStreated patients received granisetron for all 7 days of the patch application period, whereas patients received oral granisetron for 3-5 days, depending on the duration of chemotherapy. Constipation was reported less frequently than has been previously observed with intravenous $5-\mathrm{HT}_{3}$ RAs $[4,13,19]$.

The results show that the GTDS offers an effective welltolerated treatment option for CINV. Transdermal delivery of granisetron carries an important advantage over repeated oral dosing in terms of patient convenience, compliance, 
and reduced pill burden. This may be especially valuable in patients for whom swallowing is difficult or absorption of oral medications is uncertain, such as patients with previous head, neck, or gastrointestinal surgery or radiotherapy, or comorbid conditions such as xerostomia [8]. Transdermal granisetron provides additional choice for patients receiving oral chemotherapy, for whom the absence of concurrent intravenous therapies makes intravenous granisetron a less attractive alternative. The convenience of the GTDS is supported by its good adhesive properties, as demonstrated in this study. Improving patient convenience may also act to improve compliance with antiemetic therapy, with potential benefits to quality of life, overall treatment satisfaction, and compliance with future anticancer treatments [20].

Further investigation is needed to determine how the pharmacokinetics of the 5- $\mathrm{HT}_{3}$ RAs affect their pharmacodynamics; for example, it is unclear how the single-peak, sustained pharmacokinetics of the GTDS might impact on its efficacy compared to the reduced peak-tail fluctuations observed with long-acting palonosetron [11, 13]. Such investigations could also include addition of oral or intravenous granisetron to the first day of GTDS therapy, so as to achieve therapeutic levels of granisetron more rapidly. Furthermore, it would be of interest to examine the effects of combining the GTDS with NK-1 RAs, such as aprepitant. NK-1 RAs were not included in this study, as these agents were not available in some participating centers at the time of study commencement. Given the previously demonstrated efficacy of NK-1 RAs against delayed CINV when added to 5-HT 3 RA therapy [9], it would seem reasonable to postulate that their addition to GTDS therapy may improve control of CINV, particularly on the latter days of chemotherapy. The addition of NK-1 RAs to GTDS therapy would be expected to be well tolerated, as $5-\mathrm{HT}_{3} \mathrm{RAs}$ and NK-1 RAs are commonly used in combination.

The results of this randomized phase III study suggest that the GTDS could provide a valuable new option for the control of CINV in patients receiving multi-day chemotherapy regimens. Since 5-HT 3 RAs, including granisetron, are also used outside of CINV [21], the clinical application of the GTDS may eventually be broadened.

\footnotetext{
Acknowledgements The authors acknowledge the help and contribution of the following clinicians and their staff in making this study possible:

Petyo Chilingirov, Tatyana V Koynova, Hristina Markova, Mariya Racheva, Violina Taskova, Antoaneta Tomova, Hristo Tsekov, Valentina I Tzekova, and Valentina N Yanakieva (Bulgaria); Lubos Petuzelka and Jaroslav Vanasek (Czech Republic); Raju Titus Chacko, Chirag Desai, Boman Dhabhar, Raghunadharao Digumarti, Rejnish Kumar, Anish Maru, Shawnas Bahnou Noor Mohamed, Kumar Ranjan Mohapatra, Shona Nag, and Meenu Walia (India); Paula Cabrera, Jazmin Figueroa, Edwin Franco-Gonzalez, Ana Laura Rodriguez, and Evelia Sanchez (Mexico); Maciej Krzakowski, Andrzej Mruk, and Janusz Rolski (Poland); Ciprian Aldea, Florinel Badulescu, Tudor Eliade Ciuleanu, Mircea Dediu, Doina Elena Motan
}

Ganea, Lucian Miron, Delia Ruta Muresan, and Monica Patran (Romania); Kudrat M Abdulkadirov, Yulia A Alexeeva, Mikhail L Gershanovich, Nicolay V Iiyin, Elena V Karyagina, Alla S Lisyanskaya, Alfia S Nizamutdinova, Sergey V Orlov, Laslo Roman, Tatiana V Shneider, Vladimir L Vinokurov, and Andrei U Zaritskiy (Russia); Snezana Bosnjak and Dragutin Donat (Serbia); and Roberto ArevaloAraujo, Veena Charu, Ayse Dincer, Susan Ferguson, Nashat Y Gabrail, Glen R Justice, and Cameron K Tebbi (USA).

Preparation of this article was supported by ProStrakan Pharmaceuticals Inc. Assistance with writing and preparation of this article was provided by Acumen Healthcare Communications Ltd.

Funding This work was sponsored by ProStrakan Group Ltd.

Declaration Ralph Boccia has received research funding from ProStrakan Pharmaceuticals.

Lucio Gordan is an adviser to ProStrakan and has received honoraria and research funding from ProStrakan.

Steven Grunberg has acted as a consultant to ProStrakan, Helsinn, and GlaxoSmithKline, and has, in the past, received honoraria and research funding from MGI Pharma.

Julian Howell and Gemma Clark are employees of ProStrakan.

The authors declare that they have full control of the primary data and agree to allow the journal to review these data.

Open Access This article is distributed under the terms of the Creative Commons Attribution Noncommercial License which permits any noncommercial use, distribution, and reproduction in any medium, provided the original author(s) and source are credited.

\section{References}

1. Hainsworth JD (1992) The use of ondansetron in patients receiving multiple-day cisplatin regimens. Semin Oncol 19:48-52

2. De Mulder PH, Roila F, Kris MG, Marty MM (1998) Consensus regarding multiple day and rescue antiemetic therapy. Support Care Cancer 6:248-252

3. Dearnaley DP, Judson I, Root T (2002) Handbook of adult cancer chemotherapy schedules, 2nd edn. Oxon, UK, TMG Healthcare Communications, in collaboration with The Royal Marsden NHS Trust

4. Herrstedt J, Sigsgaard TC, Nielsen HA, Handberg J, Langer SW, Ottesen S, Dombernowsky P (2007) Randomized, double-blind trial comparing the antiemetic effect of tropisetron plus metopimazine with tropisetron plus placebo in patients receiving multiple cycles of multiple-day cisplatin-based chemotherapy. Support Care Cancer 15:417-426

5. Navari RM (2007) Prevention of emesis from multiple-day and highdose chemotherapy regimens. J Natl Compr Canc Netw 5:51-59

6. Lefevre G, Sedek G, Jhee SS, Leibowitz MT, Huang HL, Enz A, Maton S, Ereshefsky L, Pommier F, Schmidli H, AppelDingemanse S (2008) Pharmacokinetics and pharmacodynamics of the novel daily rivastigmine transdermal patch compared with twice-daily capsules in Alzheimer's disease patients. Clin Pharmacol Ther 83:106-114

7. Lowenthal DT, Matzek KM, MacGregor TR (1988) Clinical pharmacokinetics of clonidine. Clin Pharmacokinet 14:287-310

8. Kraut L, Fauser AA (2001) Anti-emetics for cancer chemotherapyinduced emesis: potential of alternative delivery systems. Drugs 61:1553-1562

9. Jordan K, Sippel C, Schmoll HJ (2007) Guidelines for antiemetic treatment of chemotherapy-induced nausea and 
vomiting: past, present, and future recommendations. Oncologist 12:1143-1150

10. Einhorn LH, Grunberg SM, Rapoport B, Rittenberg C, Feyer P (2010) Antiemetic therapy for multiple-day chemotherapy and additional topics consisting of rescue antiemetics and high-dose chemotherapy with stem cell transplant: review and consensus statement. Support Care Cancer. doi:10.1007/s00520-010-0920-Z [Epub ahead of print]

11. Howell J, Smeets J, Drenth HJ, Gill D (2009) Pharmacokinetics of a granisetron transdermal system for the treatment of chemotherapy-induced nausea and vomiting. J Oncol Pharm Pract 15:223-231

12. Hesketh PJ, Kris MG, Grunberg SM, Beck T, Hainsworth JD, Harker G, Aapro MS, Gandara D, Lindley CM (1997) Proposal for classifying the acute emetogenicity of cancer chemotherapy. J Clin Oncol 15:103-109

13. Eisenberg P, Figueroa-Vadillo J, Zamora R, Charu V, Hajdenberg J, Cartmell A, Macciocchi A, Grunberg S (2003) Improved prevention of moderately emetogenic chemotherapy-induced nausea and vomiting with palonosetron, a pharmacologically novel 5-HT3 receptor antagonist: results of a phase III, singledose trial versus dolasetron. Cancer 98:2473-2482

14. Gralla R, Lichinitser M, Van der Vegt S, Sleeboom H, Mezger J, Peschel C, Tonini G, Labianca R, Macciocchi A, Aapro M (2003) Palonosetron improves prevention of chemotherapy-induced nausea and vomiting following moderately emetogenic chemo- therapy: results of a double-blind randomized phase III trial comparing single doses of palonosetron with ondansetron. Ann Oncol 14:1570-1577

15. European Medicines Agency (2009) European public assessment report for Aloxi: scientific discussion. http://www.emea.europa.eu/ humandocs/PDFs/EPAR/aloxi/056305en6.pdf. Accessed March 2010

16. FDA Center for Drug Evaluation and Research (2002) Approval package for palonosetron: statistical review. http://www.access data.fda.gov/drugsatfda_docs/nda/2003/21-372_Alox_Statr.pdf. Accessed March 2010

17. Jordan K, Hinke A, Grothey A, Voigt W, Arnold D, Wolf HH, Schmoll HJ (2007) A meta-analysis comparing the efficacy of four 5-HT3-receptor antagonists for acute chemotherapy-induced emesis. Support Care Cancer 15:1023-1033

18. International Conference on Harmonisation (1998) Data analysis considerations: Analysis sets. pp 21-27

19. Handberg J, Wessel V, Larsen L, Herrstedt J, Hansen HH (1998) Randomized, double-blind comparison of granisetron versus granisetron plus prednisolone as antiemetic prophylaxis during multiple-day cisplatin-based chemotherapy. Support Care Cancer 6:63-67

20. Schnell FM (2003) Chemotherapy-induced nausea and vomiting: the importance of acute antiemetic control. Oncologist 8:187198

21. Blower PR (2003) Granisetron: relating pharmacology to clinical efficacy. Support Care Cancer 11:93-100 